\title{
Authentication of IoT device with the enhancement of One-time Password (OTP)
}

\author{
${ }^{1}$ Alice Su Wei Tang, ${ }^{2}$ Jie Hui Bong, ${ }^{3}$ Quor Ling Teh, ${ }^{4}$ Shanmugapiriya Sivalingam, ${ }^{5}$ Sharon Suet \\ Yan Chan, ${ }^{6}$ Shi Yee Khoo, ${ }^{7}$ Tahmid Mutashim Nafy \\ Faculty of Computer Science and Information Technology, Universiti Malaysia Sarawak, 94300 Kota \\ Samarahan, Sarawak, Malaysia \\ email: ${ }^{1}$ alicetsw11@gmail.com, ${ }^{2}$ huibong97@gmail.com, ${ }^{3}$ quorling@gmail.com, \\ ${ }^{4}$ shanmugapiriya95@gmail.com, ${ }^{5}$ sharon199717@gmail.com, ${ }^{6}$ shiyee530@gmail.com, 7 tmnafy95@gmail.com.
}

Date received: 24 August 2021

Date accepted: 2 October 2021

Date published: 28 October 2021

\begin{abstract}
The Robust and Energy Efficient Authentication Protocol works for Industrial Internet of Things. The Internet of Things (IoT) is an arising innovation and expected to give answers for different modern fields. The IoT enable connection of physical devices all around the world to the internet by collecting and sharing critical and real-time data among each other. The increment of devices increases the computational cost during data transmission between devices and towards the internet. In this paper we proposed a solution that is a multi-factor authentication protocol to enhance the protocol proposed by Li et al. For Industrial IoT by adding One Time Password (OTP) after the biometric information of the user is checked by the Gateway Node $(G W N)$ to be able to tackle additional network attack aside from those that are overcome by Li et al. scheme. Our contribution for this project is, we proposed the solution that a multi-factor authentication protocol to enhance the protocol proposed. For Industrial IoT by adding One Time Password (OTP) after the biometric information of the user is checked by the Gateway Node $(G W N)$ to be able to tackle additional network attack aside from those that are overcome. The idea of adding OTP is inspired by where they scheme correlates to biometric of user as well. Our proposal is lower cost than the three protocols regarding authentication overhead and computational cost perspectives. Challenges and future directions of this paper examined the security shortcomings of a client confirmation convention for WSN, which is as proposed by Chang and Le. To address the normal security shortcomings of past protocols, we proposed a strong and energy effective three-factor authentication protocol for WSN.
\end{abstract}

Keywords: IoT, Multi-factor authentication, One-time password, WSN

Copyright: This is an open access article distributed under the terms of the CC-BY-NC-SA (Creative Commons AttributionNonCommercial-ShareAlike 4.0 International License) which permits unrestricted use, distribution, and reproduction in any medium, for non-commercial purposes, provided the original work of the author(s) is properly cited.

\section{Introduction}

Mutual authentication and specifically multifactor authentication mechanism is not a new research paradigm. Several researchers have proposed several methods to ensure secure authentication mechanisms in any communication protocols (Khan et al., 2021) (Khan et al., 2019) (Maikol et al., 2020) (Khan et al., 2015) (Balan et al., 2018). The Internet of Things (IoT) is an arising innovation and expected to give answers for different modern fields. IoT is referring to the connection between millions and billions of physical devices that allows sharing and collecting of data (Ranger, 2020). (WSN) wireless sensor networks as one of the essential innovations of the IoT that can be utilized to gather the necessary climate boundaries for explicit applications. There are three sorts of members in WSN; they are users, sensor nodes and gateway. Sensor nodes have restricted calculation, power sources and gateway. Two authentication protocols for WSN and they are safer with amazing forward mystery another one is lightweight. They are comparable, and the two plans contain of three stages, and they are password change, registration, and authentication (Li et al., 2018).

The problem statement is that the IoT enable connection of physical devices all around the world to the internet by collecting and sharing critical and real-time data among each other (Gope \& Sikdar, 2019). The increment of devices increases the computational cost during data transmission between devices and towards the internet. This 
gives rise to network congestion as the algorithms of encryption and number of operations performed in each session escalates with the number of devices. Moreover, as the IoT devices are deployed in open and public spaces of the internet, it is more prone for adversary to deploy internal and external attacks towards the network such as Impersonation attack, spoofing, Replay attack etc (Gope \& Sikdar, 2019) (Varanasi, 2020). It is needed to propose a multifactor authentication scheme for IoT that involved lower computational cost and authentication cost plus increase of network security assurance for the users.

Thus, our proposed solution to address the problem statement stated is a multi-factor authentication protocol to enhance the protocol proposed by Li et al. ( $\mathrm{Li}$ et al., 2018). The ideation of adding OTP is inspired by Imran et al. (2017) where their scheme correlates to the biometric information of user as well. Method to solve the problem is mainly on the Authentication phase where the OTPi sent from the Gateway for the user device to enter and sent back to Gateway Node (GWN) to counter check (Imran et al., 2017). The OTP is only sent to the device that request for login access and it is only available for one login session, thus it is impossible to have the same OTPi used for different session (Digital Guide Ionos, 2020).

Our contribution for this project is to propose a multi-factor authentication protocol to enhance the protocol proposed by $\mathrm{Li}$ et al. (Li et al., 2018). For Industrial IoT by adding One Time Password (OTP) after the biometric information of the user is checked by the GWN to be able to tackle additional network attack aside from those that are overcome. The attacks that are able to be mitigated from the enhancement of the scheme should overcome attacks that is to be discussed in Section V.

Aside from that, computational and authentication cost of the proposed scheme are considered as well to ensure the proposed scheme is lightweight yet brings secure for IoT devices. Three other proposed schemes are taken for comparison with our proposed scheme and more in depth explanation is in Section VI

Furthermore, this paper examined the shortcomings in security of a client confirmation convention for WSN proposed by Chang and Le. It is presented in the examination that it able to forestall normal attacks at most time and gives some ideal usefulness. In the interim, the security and execution correlations show that the proposed protocol is strong than other comparable conventions with low computational cost, decreases the power consumption and lightweight. Take IoT-based brilliant medical services for instance, the plan of lightweight confirmation convention for asset restricted wearable gadgets, protection saving clinical information total plan, and security saving clinical information distributing are open issues (Sethia et al., 2018). The proposed work can be extended to enhance end to end security mechanism in network intrusion detection system or malware detection system (Ahmad el al., 2021; Dildar et al., 2017; Khan et al., 2021; Khan et al.2017).

\section{Related Work}

In these recent years, communication between different IoT devices in various domain have been secured with the implementation of authentication. Such implemented are introduced and constantly proposing an enhancement of authentication in various work.

Sethia et. al (2018) presented a unique structure aimed at the NFC Secure Element (SE)-centred attestation and mutual authentication on behalf of IoT admission utilizing NFC-based Host Card Emulation (HCE) style with an end-client gadget such as a portable device. Near field communication (NFC) is a small-scale wireless machinery that allows electronic devices to communicate easily and securely within a short distance that is only a few centimeters (Christensson, 2019). Their proposed scheme provides a developer-friendly platform and even support mutual communication and large amounts of storage, as well as other NFC modes. It uses an asymmetric algorithm - RSA, and symmetric algorithm - AES encryption algorithms.

Feng (2020) enhanced the security protocol from Safkhani and Vasilakos (2019) to overcome the drawbacks of security problems in RFID for healthcare system such as impersonation attacks, traceability attacks, replay attacks, desynchronization attacks, time measurement attack and secret disclosure attacks (Feng, 2020). Mutual authentication, message of tag in two different sessions, adding in random number etc. are the implementation introduced in Feng proposed protocol. However, the proposed protocol possesses higher computational cost despite lower risk of encountering network attack.

Banerjee et al. (2019) proposed a robust lightweight anonymous authentication protocol for IoT ecosystem that is able to counter new attack like device impersonation. Their proposed protocol based on lightweight operations such as "Physically Unclonable Functions (PUFs)", "fuzzy extractor functions", "one-way hash functions", and "bitwise XOR operations" (Banerjee et al., 2019). The PUFs are intended to match an entry only to an output based on a computer's physical microstructure. Each PUF circuit has its own challenge-response pair, which is an input-output pair. While a high degree of reliability of a PUF can be established, noise in PUF remains a significant problem. Therefore, fuzzy extractor is used to overcome the problem. The fuzzy extractor is affected by two approaches, the probabilistic Gen $(\cdot)$ function and the deterministic Rep ( function). One-way hash functions for data integrity are extensively used. Cryptographic one-way hash functions are engineered to be extremely sensitive to even minor changes in the input. The proposed scheme requires the user $U$, the gateway node GN, and the smart system SD to execute during the login and authentication process. 
Gope and Sikdar (2019) stated that IoT devices are often installed in open and public spaces, making them susceptible to physical attack and the cloning attack. Traditional password or a hidden key using an authentication system, in which a mutual secret is the only authentication factor, are insufficient to solve the security issues in these scenarios. He proposed that any attempt to tamper with the computer, on the other hand, changes the actions of the PUF embedded in it, rendering the PUF useless. Aside from that, his proposed protocol also employs the key-hash feature and the challenge-response principle to not allow the adversary to attack. Plus, PUFs are resistant to cloning which then makes it secure against the specific attack.

Cao et al. (2019) work on access authentication and distribution protocol with aggregation signcryption with no bilinear pairings in the $5 \mathrm{G}$ network and it is also applicable in LTE network. However, it is not resistant towards Quantum attack. By enhancing their previous proposed protocol, in Cao et al. (2019), an addition of lattice-based homomorphic encryption is used. Encrypted messages from NB-IoT devices are sent using the public key of AMF (access and mobility manage function) during the transmission. It will be able to mitigate MiM attack. This is due to private key of AMF is needed to decrypt the message. Aggregation signcryption (signature encryption) was used to mitigate DoS attack which also contributes towards avoidance of network congestion. Quantum attack that occurs in the previous proposed schemes by Cao et al. (2019) is mitigated as well by the usage of private key for decryption that disable the attacker to forge signcryption.

Li et al. (2020) designed SLAKA by applying the cryptographic hash function, the blurry extractor method, and the XOR operations. It will be able to overcome attacks which are stolen or lost mobile terminal attacks, stolen or lost wearable device attacks, man-in-the-middle attacks, password change attacks, user impersonation attacks, wearable device impersonation attacks, and replay attacks. SLAKA uses timestamps during the message transmission that is good in countering replay attack. Computational and communication costs of SLAKA protocol is lower than the other existing schemes that are proposed in other work as comparison.

Li et al. (2018) proposed a strong and energy effective three-factor authentication protocol for WSN that involves with user, password and biometric. Examination shows it can forestall most normal attacks and gives some ideal usefulness. In the interim, the security and execution correlations show that the proposed protocol is strong than other comparable conventions with low computational cost, decreases the power consumption and lightweight. Hence, their proposed protocol is vigorous furthermore, proficient for IoT applications. IoT advancements can be utilized in numerous fields, for example, brilliant network and keen medical services, and there are numerous securities also, security insurance issues to be settled. Take IoT-based brilliant medical services for instance, the plan of lightweight confirmation convention for asset restricted wearable gadgets, protection saving clinical information total plan, and security saving clinical information distributing are open issues.

\section{Review Li et. Al Scheme}

In this paper, we will be enhancing the proposed scheme stated by Li et al. (2018). Figure 1 below presents the current scheme that is proposed by Li et al. (2018). From this scheme, we will be adding in OTP during the authentication phase between the user and the gateway.

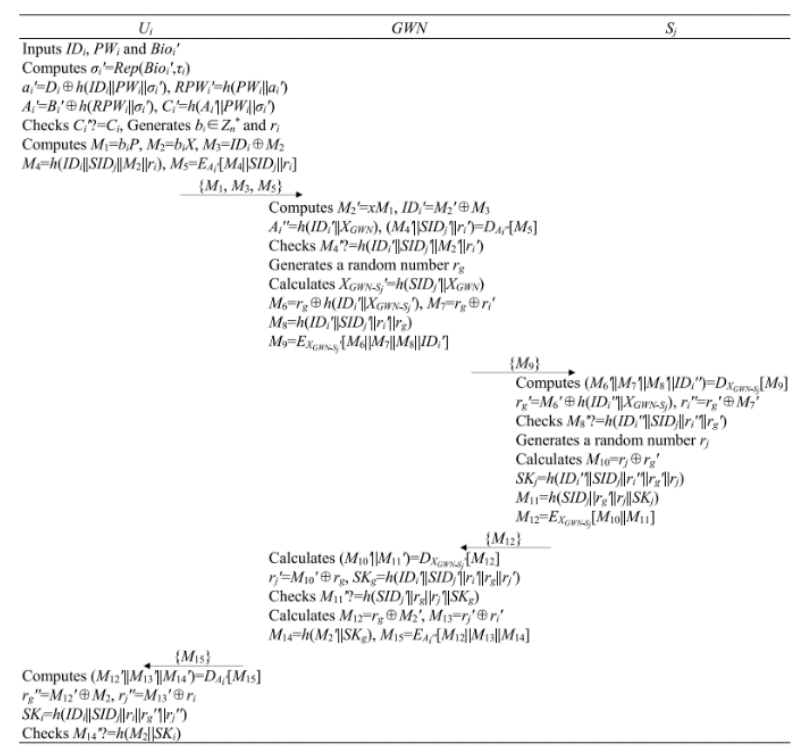

Figure 1 : Proposed scheme by Li et al. (2018) 


\section{Proposed Solution}

For this section, we have proposed a multi-factor authentication protocol to enhance the protocol proposed by Li et al. (2018) for Industrial IoT by adding One Time Password (OTP) after the biometric information of the user is checked by the Gateway Node (GWN) to be able to tackle additional network attack aside from those that are overcome by Li et al. (2018) scheme. The ideation of adding OTP is inspired by Imran et al. (2017) where they scheme correlates to identifying biometric information of user as well. Table 1 display the notation that is used in the protocol.

Table 1 : Notations

\begin{tabular}{|c|c|}
\hline Notation & Description \\
\hline $\mathrm{U}_{\mathrm{i}}$ & User \\
\hline $\mathrm{ID}_{\mathrm{i}}$ & Corresponding identity \\
\hline $\mathrm{PW}_{\mathrm{i}}$ & Password \\
\hline $\mathrm{Bio}_{\mathrm{i}}$ & Biometric information \\
\hline$a_{i}, r_{j}$ & Random number \\
\hline $\mathrm{RPW}_{\mathrm{i}}$ & Masked password \\
\hline GWN, $X_{\mathrm{GWM}}$ & Gateway node and its master secret key \\
\hline $\mathrm{S}_{\mathrm{j}}, \mathrm{SID}_{\mathrm{j}}$ & The sensor node and its identity \\
\hline $\mathrm{OTP}_{\mathrm{i}}$ & One Time Password \\
\hline $\mathrm{X}_{\mathrm{GWN}-\mathrm{Ui}}$ & Secret key shared between GWN and $U_{i}$ \\
\hline TSM & Timestamp \\
\hline $\mathrm{X}_{\mathrm{G}}$ & Secret key only known by gateway \\
\hline $\mathrm{X}_{\mathrm{GW}}$ & Gateway key that is shared with user \\
\hline
\end{tabular}

\subsection{Initiation Phase}

During initialization of the system, a long term secret key chosen by GWN, $\mathrm{X}_{\mathrm{GWN}}$, hash function as $\mathrm{h}(\cdot)$, implementing symmetric cryptography that includes encryption and decryption algorithm labelled $\mathrm{E}_{\mathrm{k}}(\cdot)$ and $\mathrm{D}_{\mathrm{k}}(\cdot)$ respectively.

Apart from that, elliptic curve E selected by GWN is dependant with the finite field Fp. Then, an order of large prime $\mathrm{n}$ by subgroup $\mathrm{G}$ of $\mathrm{E}$ is chosen by GWN while point $\mathrm{P}$ is the generator. Afterwards, private key represent by $x$ is bring out by GWN and the corelated public key $\mathrm{X}$ is calculated, where $\mathrm{x} \in \mathrm{Z}_{\mathrm{n}}{ }^{*} \mathrm{n}$ and $\mathrm{X}=x P$. Lastly, $x$ is being kept secretly by GWN and parameters $\left\{\mathrm{E}\left(\mathrm{F}_{\mathrm{p}}\right), \mathrm{G}, \mathrm{P}, \mathrm{X}\right\}$ are produced.

For initializing sensor network, sensor node $S_{j}$ has its identity SID $_{j}$ selected by GWN. After that, the shared secret key $\mathrm{X}_{\mathrm{GWN}-\mathrm{Sj}}=\mathrm{h}\left(\mathrm{SID}_{\mathrm{j}} \| \mathrm{X}_{\mathrm{GWN}}\right)$ is calculated by GWN. $\mathrm{X}_{\mathrm{GWN}-\mathrm{Sj}}$ is kept by GWN into the memory of $\mathrm{S}_{\mathrm{j}}$, and the target area will be having sensor nodes deployed in it.

\subsection{Registration Phase}

For the user to be recognized being an eligible user for the system, the mentioned steps below are to be conducted between the user $U_{i}$ and the gateway node GWN. By completing the registration phase, it will result of allowing the $\mathrm{U}_{\mathrm{i}}$ to access the sensor data through their mobile devices according to real-time.

Step 1: $\mathrm{U}_{\mathrm{i}}$ chooses an identity $\mathrm{ID}_{\mathrm{i}}$, a password $\mathrm{PW}_{\mathrm{i}}$ and a random number. In the meanwhile, Biometric information $\mathrm{Bio}_{\mathrm{i}}$ will be provided by $\mathrm{U}_{\mathrm{i}}$ and extracted with fuzzy extractor [1] through their mobile device, alongside $\operatorname{Gen}\left(B_{i o}\right)=\left(\sigma_{i}, \tau_{i}\right)$ is obtained. Next, masked password $\mathrm{RPW}_{\mathrm{i}}=\mathrm{h}\left(\mathrm{PW}_{\mathrm{i}} \| \mathrm{a}_{\mathrm{i}}\right)$ is calculated by $\mathrm{U}_{\mathrm{i}}$, $\left\{\mathrm{ID}_{\mathrm{i}}, \mathrm{RPW}_{\mathrm{i}}, \sigma_{\mathrm{i}}\right\}$ is submitted for GWN to register $\mathrm{U}_{\mathrm{i}}$ mobile device.

Step 2: When registration request from $U_{i}$ is received, GWN will first examine whether ID $_{i}$ is in the database. If yes, a new identity will be asked from $U_{i}$ for submission. Else, $A_{i}=h\left(I_{i} \| X_{G W N}\right)$ and $B_{i}=$ $\mathrm{A}_{\mathrm{i}} \oplus \mathrm{h}\left(\mathrm{RPW} \mathrm{W}_{\mathrm{i}} \| \sigma_{\mathrm{i}}\right)$ will be computed by GWN, and GWN sends $\left\{\mathrm{B}_{\mathrm{i}}, \mathrm{E}_{\mathrm{k}}(\cdot), \mathrm{D}_{\mathrm{k}}(\cdot), \mathrm{X}\right\}$ to $\mathrm{U}_{\mathrm{i}}$ through an authentic manner.

Step 3: After obtaining the parameters sent by GWN, $U_{i}$ determines $A_{i}=B_{i} \oplus h\left(R P W_{i} \| \sigma i\right), C_{i}=h\left(A_{i}\left\|P W_{i}\right\|\right.$ $\left.\sigma_{i}\right), D_{i}=a_{i} \oplus h\left(I_{i}\left\|P W_{i}\right\| \sigma_{i}\right)$, and stores $\left\{B_{i}, C_{i}, D_{i}, E_{k}(\cdot), D_{k}(\cdot)\right.$, Gen $\left.(\cdot), \operatorname{Rep}(\cdot), X, \tau_{i}\right\}$ into mobile devices of $U_{i}$

\subsection{Authentication and Key Agreement Phase}

Whenever there is a need for $U_{i}$ to access $S_{j}$ sensor data, with the additional of OTP $P_{i}$ during the authentication process, $\mathrm{U}_{\mathrm{i}}, \mathrm{GWN}$, and $\mathrm{S}_{\mathrm{j}}$ performed the stated authentication steps as listed below. By the end of authentication 
phase, a session key is shared among these three parties. Figure 1 presents the processes that is implemented during this phase.

Step 1: $U_{i}$ uses their mobile devices inputting $\mathrm{ID}_{i}$ and $\mathrm{PW}_{i}$ plus have their biometric information, Bio $i$ imprinted with fuzzy extractor. Then $\sigma_{i}^{\prime}=\operatorname{Rep}\left(B_{i o}^{\prime}, \tau_{i}\right), a_{i}^{\prime}=D_{i} \oplus h\left(\mathrm{ID}_{i}\|\mathrm{PW}\|_{i} \| \sigma_{i}^{\prime}\right), R P W_{i}^{\prime}=$ $h\left(P W_{i} \| a_{i}^{\prime}\right), A_{i}^{\prime}=B_{i}^{\prime} \oplus \mathrm{h}\left(R P W_{i}^{\prime} \| \sigma_{i}^{\prime}\right), C_{i}^{\prime}=h\left(A_{i}^{\prime}\left\|P W_{i}\right\| \sigma_{i}^{\prime}\right)$ is calculated by the mobile devices, and checks $C_{i} \stackrel{?}{=} C_{i}$. If there is more than one out of the three factors mentioned is invalid, login request will be refuse by the mobile device. Else, next step will be performed. Two random numbers is produced, $b_{i} \in$ $Z_{n}^{*}$ and $r_{i}$, and computes $M_{1}=b_{i} P, M_{2}=b_{i} X, M_{3}=\mathrm{ID}_{i} \oplus M_{2}, M_{4}=h\left(\operatorname{ID}_{i}\left\|\operatorname{SID}_{j}\right\| M_{2} \| r_{i}\right)$ and $M_{5}=$ $E_{A_{i}^{\prime}}\left[M_{4}\left\|S I D_{j}\right\| r_{i}\right]$. The login request $\left\{M_{1}, M_{3}, M_{5}, \mathrm{TSM}_{1}\right\}$ to $\mathrm{GWN}$ is then being submitted from the mobile device.

Step 2: Upon login request is received, GWN calculates $M_{2}=x M_{1}, \mathrm{ID}_{i}=M_{2}^{\prime} \oplus M_{3}, A_{i}=h\left(\mathrm{ID}_{i} \| X_{\mathrm{GWN}}\right),($ $\left.M_{4}^{\prime}\left\|S I D_{j}\right\| r_{i}^{\prime}\right)=D_{A_{i}^{\prime \prime}}\left[M_{5}\right]$, and checks $M_{4}^{\prime} \stackrel{?}{=} h\left(\operatorname{ID}_{i} \operatorname{SID}_{j} M_{2} r_{i}\right) . \mathrm{TSM}_{1}$ is checked to ensure real-time transmission as it can be evaluated that is it being attacked by the adversary when there is a delay. GWN will stop the login request when the equation does not hold. Otherwise, GWN generates the OTP . OTP $_{u}$ is generated by $\mathrm{z}_{\mathrm{u}}=\mathrm{x}_{\mathrm{u}} \oplus \mathrm{OTP}_{\mathrm{u}}, \mathrm{x}_{\mathrm{u}}=\mathrm{h}\left(\mathrm{ID}_{\mathrm{i}} \| \mathrm{X}_{\mathrm{GWN}-\mathrm{Ui}}\right), \mathrm{y}_{\mathrm{u}}=\mathrm{h}\left(\mathrm{BiO}_{\mathrm{i}} \| \mathrm{X}_{\mathrm{GWN}-\mathrm{Ui}}\right)$. GWN then sends back $<\mathrm{z}_{\mathrm{u}}$, $\mathrm{x}_{\mathrm{u}}, \mathrm{y}_{\mathrm{u}}, \mathrm{TSM}_{2}>$ to $\mathrm{U}_{\mathrm{i}}$.

Step 3: $\mathrm{U}_{\mathrm{i}}$ extract $\mathrm{OTP}_{\mathrm{u}}=\mathrm{x}_{\mathrm{u}} \oplus \mathrm{yu}$ and send back the $\mathrm{OTP}_{\mathrm{u}}$ received by the GWN with $\mathrm{e}_{\mathrm{u}}=\mathrm{OTP}_{\mathrm{u}} \oplus \mathrm{y}_{\mathrm{u}}$

Step 4: When GWN receives e from $U_{i}$ mobile device, GWN extract OTP ${ }_{u}^{*}=\mathrm{e}_{\mathrm{u}} \oplus$ yu and Checks $\mathrm{OTP}_{\mathrm{u}}{ }^{*}$ $\stackrel{?}{=} \mathrm{OTP}_{\mathrm{u}}$. If the $\mathrm{OTP}_{\mathrm{u}}$ * obtained is the same as $\mathrm{OTP}_{\mathrm{u}}$, a random number $r_{\mathrm{g}}$ is generated by GWN, and calculates $\quad X_{G W N-S_{j}}^{\prime}=h\left(S I D_{j}^{\prime} \| X_{G W N}\right), \quad M_{6}=r_{g} \oplus \mathrm{h}\left(I D_{i}^{\prime} \| X_{G W N-S_{j}}^{\prime}\right), \quad M_{7}=r_{g} \oplus r_{i}^{\prime}, \quad M_{8}=$ $h\left(I D_{i}^{\prime}\left\|S I D_{j}^{\prime}|| r_{i}^{\prime}\right\| r_{g}\right), M_{9}=E_{X_{G W N-S_{j}}^{\prime}}\left[M_{6}\left\|M_{7}\right\| M_{8} \| I D_{i}^{\prime}\right]$. At last, GWN sends $\left\{M_{9}\right\}$ to $S_{j}$.

Step 5: When $\mathrm{S}_{\mathrm{j}}$ receives messages from GWN, $\left(M_{6}^{\prime}\|\| M^{\prime}{ }_{7}\left\|M_{8}^{\prime}\right\| \mathrm{ID}_{i}^{\prime}\right)=D_{X_{G W N-S_{j}}}\left[M_{9}\right], r_{g}^{\prime}=$ $M_{6}^{\prime} \oplus h\left(I D_{i}^{\prime \prime} \| X_{G W N-S_{j}}\right), r_{i}^{\prime \prime}=r_{g}^{\prime} \oplus M_{7}^{\prime}$ is calculated by $\mathrm{S}_{\mathrm{j}}$ and checks $M_{8}^{\prime} \stackrel{?}{=} h\left(I D_{i}^{\prime \prime}\left\|\operatorname{SID}_{j}\right\| r_{i}^{\prime \prime} \| r_{g}^{\prime}\right)$. If $M_{8}^{\prime} \stackrel{?}{=} h\left(I D_{i}^{\prime \prime}\left\|\operatorname{SID}_{j}\right\| r_{i}^{\prime \prime} \| r_{g}^{\prime}\right)$ is not equivalent, the termination of session is conducted. If not, $S_{j}$ generates a random number $r_{j}$, and calculates $M_{10}=r_{j} \oplus r_{g}^{\prime}, S K_{j}=h\left(I D_{i}^{\prime \prime}\left\|\operatorname{SID}_{j}\right\| r_{i}^{\prime \prime}\left\|r_{g}^{\prime}\right\| r_{j}\right)$, $M_{11} \stackrel{?}{=} h\left(\operatorname{SID}_{j}\left\|r_{g}^{\prime}\right\| r_{j} \| S K_{j}\right)$ and $M_{12}=E_{X_{G W N-S}}\left[M_{10} \| M_{11}\right]$. Then $\mathrm{S}_{\mathrm{j}}$ responses $\left\{\mathrm{M}_{12}\right\}$ to GWN.

Step 6: Upon receiving response message by $S_{j},\left(M^{\prime}{ }_{10} \| M^{\prime}{ }_{11}\right)=D_{X^{\prime}}{ }_{G W N-S_{j}}\left[M_{12}\right], r_{j}^{\prime}=M^{\prime}{ }_{10} \oplus r_{g}$, $S K_{g}=h\left(\operatorname{ID}_{i}^{\prime}\left\|\operatorname{SID}_{j}^{\prime}\right\| r_{i}^{\prime}\left\|r_{g}\right\| r_{j}^{\prime}\right)$ is calculated by GWN, $M^{\prime}{ }_{11} \stackrel{?}{=} h\left(\operatorname{SID}_{j}^{\prime}\left\|r_{g}\right\| r_{j}^{\prime} \| S K_{g}\right)$ is checked afterwards. If they are not equal, the session will be terminated. Otherwise, GWN calculates $M_{12}=r_{g} \oplus$ $M_{2}^{\prime}, M_{13}=r_{j}^{\prime} \oplus r_{i}^{\prime}, M_{14}=h\left(M_{2}^{\prime} \| S K_{g}\right)$, and $M_{15}=E_{A^{\prime} i^{\prime}}\left[M_{12}\left\|M_{13}\right\| M_{14}\right]$. Then, GWN submits $\left\{\mathrm{M}_{15}\right\}$ to $\mathrm{U}_{\mathrm{i}}$.

Step 7: When receiving $\left\{M_{15}\right\}$ from GWN, $U_{i}$ calculates $\left(M_{12}^{\prime}\left\|M_{13}^{\prime}\right\| M_{14}^{\prime}\right)=D_{A_{i}^{\prime}}\left[M_{15}\right], r_{g}^{\prime \prime}=M_{12}^{\prime} \oplus$ $M_{2}, r_{j}^{\prime \prime}=M_{13}^{\prime} \oplus r_{i}, S K_{i}=h\left(I D_{i}\left\|S I D_{j}\right\| r_{i}\left\|r_{g}^{\prime \prime}\right\| r_{j}^{\prime \prime}\right)$, and $M_{14}^{\prime} \stackrel{?}{=} h\left(M_{2} \| S K_{i}\right)$. Termination of session is conducted if it is not equal. Else, mutual authentication is achieved among $\mathrm{U}_{\mathrm{i}}, \mathrm{GWN}$ and $\mathrm{S}_{\mathrm{i}}$, and a shared session key $S K_{i}\left(=S K_{g}=S K_{j}\right)$ is provided between these three parties to ensure continuous communication yet secure.

Figure 2 exhibit the process during the registration phase and authentication and key agreement phase. 


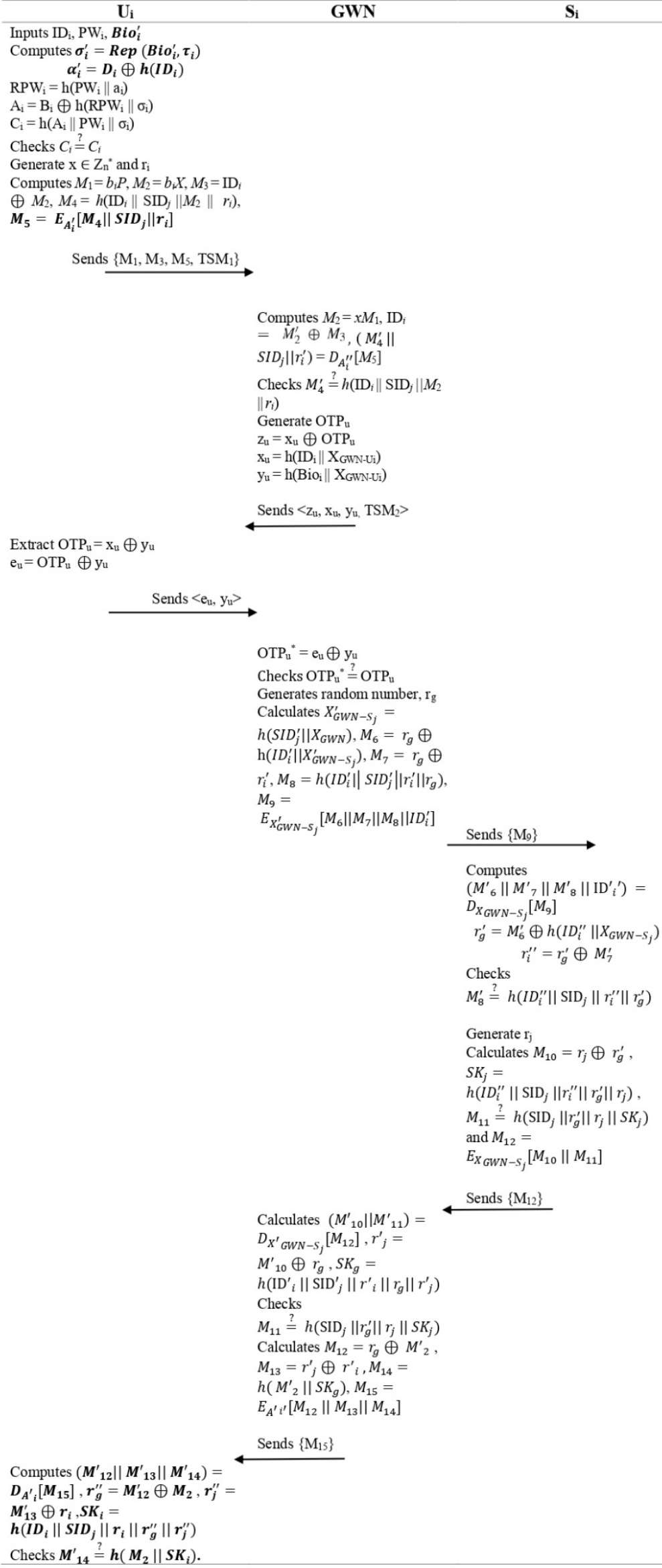

Figure 2: Proposed scheme diagram 


\subsection{Password Change Phase}

In an event where the user forgotten or wanted to renew their static password, this phase allows them to change the password. The users able to change their password without sending any request to the GWN.

$\mathrm{U}_{i}$ utilized their mobile devices and inputs $\mathrm{ID}_{i}, \mathrm{PW}_{i}$, and imprints the biometric information $B i o_{i}^{\prime}$ with fuzzy extractor. Next, a request of password change is sent by the user. The mobile device from user then calculates $\sigma_{i}^{\prime}=\operatorname{Rep}\left(B_{i}^{\prime}, \tau_{i}\right), a_{i}^{\prime}=\mathrm{D}_{i} \oplus h\left(\mathrm{ID}_{i}\left\|\mathrm{PW}_{j}\right\| \sigma_{i}^{\prime}\right), R P W_{i}^{\prime}=h\left(\mathrm{PW}_{i} \| a_{i}^{\prime}\right), A_{i}^{\prime}=B_{i}^{\prime} \oplus h\left(R P W_{i}^{\prime} \| \sigma_{i}^{\prime}\right)$, and $C_{i}^{\prime}=$ $h\left(A_{i}^{\prime}\left\|\mathrm{PW}_{i}\right\| \sigma_{i}^{\prime}\right)$, and checks $C_{i}^{\prime} \stackrel{?}{=} \mathrm{C}_{i}$. If one of the three factors is invalid from the user's input, the user's mobile device will decline the request for password change. Otherwise, $\mathrm{U}_{i}$ a new password $P W_{i}^{\text {new }}$ can be filled in by $\mathrm{U}_{i}$. In the end, $C_{i}^{\text {new }}=h\left(A_{i}^{\prime}\left\|P W_{i}^{\text {new }}\right\| \sigma_{i}^{\prime}\right), D_{i}^{\text {new }}=a_{i}^{\prime} \oplus h\left(\mathrm{ID}_{i}\left\|P W_{i}^{\text {new }}\right\| \sigma_{i}^{\prime}\right)$ is calculated by the $\mathrm{U}_{\mathrm{i}}$ mobile device and replaces $\left\{\mathrm{B}_{i}, \mathrm{C}_{i}, \mathrm{D}_{i}\right\}$ with $\left\{B_{i}^{\text {new }}, C_{i}^{\text {new }}, D_{i}^{\text {new }}\right\}$.

\section{Security Analysis}

Through the improvement by adding in timestamps and OTP into the protocol, we were able to analyze the types of network attack that can be mitigated and being more secure from adversary or ill-intention people. This section provides a clearer picture on how the improvements and computational processes is implemented in the protocol to mitigate attacks.

\subsection{Quick Detection for Unauthorized login}

Three factors are used in the proposed protocol to ensure authentication implemented is appropriate. If the verification of user's biometric, identity, and password passed, the sensor data may only be accessed by the user. Mobile device will be able to find out and decline unwanted logins triggered by incorrect password with the error password checking mechanism. This verification procedure involved with three-factor is in AKA phase step 1.

\subsection{Proper Mutual Authentication}

GWN and $U_{i}^{\prime}$ 's mutual authentication is based on the shared secret information $A_{i}=h\left(\mathrm{ID}_{i} \| X_{G W N}\right)$ and $M_{2}=b_{i} x P$, where $M_{2}$ is created by $U_{i}$. $A_{i}$ may be obtained from $B_{i}$ and $D_{i}$ using identity, password, and biometric data. GWN may obtain $M_{2}$ and $A_{i}$ from $U_{i}$ 's login request message $\left\{M_{1}, M_{3}, M_{5}, T S M_{1}\right\}$ using x. GWN and $U_{i}$ may authenticate each other using the shared secret information $A_{i}$ and $M_{2}$.

The shared secret key $=h\left(\operatorname{SID}_{j} \| X_{G W N}\right)$, which is kept in $S_{j}$ 's memory and may be obtained by GWN when it receives $\operatorname{SID}_{j}$ via the login request message, is used for mutual authentication between GWN and $S_{j}$. GWN appear to be a trustworthy towards sensor nodes and users in the proposed protocol. If both $U_{i}$ and $S_{j}$ are authenticated by GWN, they think each other is legitimate.

\subsection{User Anonymity and Untraceability}

Assume that $A$ intercepts the login request message $\left\{M_{l}, M_{3}, M_{5}\right\}$, where $M_{l}=b_{i} P, M_{2}=b_{i} X, M_{3}=\mathrm{ID}_{i} \oplus M_{2}, M_{4}$ $=h\left(\mathrm{ID}_{i}\left\|\mathrm{SID}_{j}\right\| M_{2} \| r_{i}\right)$, and the two random numbers $b_{i} \in$ and $r_{i}$. It is noticeable that without knowing $\mathrm{x}, A$ unable to expose $\mathrm{ID}_{i}$ through the login request message, thus achieved user anonymity with the suggested protocol. GWN, on the other hand, may retrieve $U_{i}$ 's identification $\mathrm{ID}_{i}$ by utilizing $\mathrm{x}$, user identification verification is enabled by our proposed protocol. Furthermore, with the variability of random numbers $b_{i} \in$ and $r_{i}$, dynamic changes take place for each of the element in login request message. As a result, $A$ is unable to track a specific user over the public channel.

\subsection{Resist Mobile Device Loss Attack}

Mobile device loss attack is when through the exploitation of one's devices such as tablets and smartphones to get information or to download malicious application (Ismail et al., 2017). If $A$ obtains $U_{i}$ 's lost mobile device, $A$ can use power analysis attacks to extract the parameters $\left\{B_{i}, C_{i}, D_{i}, E_{k}(\cdot), D_{k}(\cdot), \operatorname{Gen}(\cdot), \operatorname{Rep}(\cdot), X, \tau_{i}\right\}$ from the device. $B_{i}=h\left(\mathrm{ID}_{i} \| X_{G W N}\right) \oplus h\left(h\left(\mathrm{PW}_{i} \| a_{i}\right) \| \sigma_{i}\right), C_{i}=h\left(h\left(\mathrm{ID}_{i} \| X_{G W N}\right)\left\|\mathrm{PW}_{i}\right\| \sigma_{i}\right), D_{i}=a_{i} \oplus h\left(\mathrm{ID}_{i}\left\|\mathrm{PW}_{i}\right\| \sigma_{i}\right)$, and $U_{i}$ 's biometric data extracts a random string $\sigma_{i}$. Since $B_{i}, C_{i}$ and $D_{i}$ are computed from at least two of three unknown lengthy random strings $X_{G W N}, a_{i}$ and $\sigma_{i}$, the parameters retrieved from the mobile device by an attacker $A$ will not be able to correctly determine the identity and password. Hence, it will prevent mobile device loss attack 


\subsection{Resist Impersonation Attack}

According to the AKA phase description, attacker require the $\mathrm{ID}_{i}$ and $A_{i}=h\left(\mathrm{ID}_{\mathrm{i}} \| x\right)$ 's information in order to impersonate a trusted user and produce a successful login request. However, based on the description on user anonymity and untraceability, the proposed protocol ensures user anonymity, with no party other than GWN being able to get the user's identity $\mathrm{ID}_{\mathrm{i}}$. According to the AKA phase, $A_{i}$ can also be computed by GWN using $\mathrm{x}$ when he/she obtains $\mathrm{ID}_{i}$ or obtained by $U_{i}$ from $B_{i}$ with the presence of $\mathrm{PW}_{i}, a_{i}$ and $\sigma_{i}$. As a result, attacker $A$ will be unable to get $A_{i}$ without possessing the necessary information, and our protocol will be able to prevent a user impersonation attack. Furthermore, $\mathrm{x}$ and $\mathrm{X}_{\mathrm{GWN}}$ as the secret keys are needed for an attacker to produce legitimate communication messages to impersonate the GWN. However, GWN is the only one who knows about $\mathrm{x}$ and $\mathrm{X}_{\mathrm{GWN}}$, therefore the suggested protocol can prevent a gateway impersonation attack.

\subsection{Resist Replay Attack}

To prevent replay attacks, the suggested protocol uses a random number method. $U_{i}, S_{j}$ and GWN, respectively, produce random numbers $b_{i} \in, r_{i}, r_{g}$ and $r_{j}$ in each session of the AKA phase to calculate the communication messages. Because the random numbers change constantly with each session, and the fresh random numbers ensure the freshness of the communication in each session. As a result, our protocol is protected against replay attacks. The timestamp used during the login request sent from user and the OTP sent from gateway able to counter this attack as well as if the messages is sent and received longer than as expected, it indicates that the adversary trying to get the message packets through the network and alter the contents of the messages (Kaspersky, 2021).

\subsection{Sensor Node Anonymity}

The identification of the sensor node is not sent in plaintext across the public channel in the proposed protocol. Encryption of $S_{j}$ 's identity $\mathrm{SID}_{j}$ in the login request message $M_{5}$ is done and enables retrieval of GWN with x from $\mathrm{M}_{5}$ when $U_{i}$ wishes to access $S_{j}^{\prime}$ s sensor data. Any attacker who does not know x is unable to get the identity of a sensor node, $\mathrm{SID}_{j}$, thus the proposed protocol achieves sensor node anonymity.

\subsection{Friendly Password Change}

The proposed protocol enables the mobile device to rapidly identify illegal logins caused by incorrect passwords and allows users to renew their passwords once the mobile device has validated their identification, biometrics, and earlier password. As a result, password change phase in the proposed protocol is user-friendly, and it is stated in the password change phase.

\subsection{Resist Against Spoofing Attack}

Spoofing is the where the adversary imitates or impersonates a trusted contact or brand to obtain one's sensitive information (Belcic \& Farrier, 2021). The use of OTP can counter this attack as the adversary will be unable to login although they obtained the user's login details. Upon receiving the login details from the user $U_{i}$, the gateway will send out the OTP ${ }_{u}$ back to the user for them to insert accordingly through their mobile devices. The gateway will then counter check whether the OTP ${ }_{u}$ received from the mobile device is the same as what is sent out by $\mathrm{OTP}_{\mathrm{u}}{ }^{*} \stackrel{?}{=} \mathrm{OTP}_{\mathrm{u}}$, where $\mathrm{OTP}_{\mathrm{u}}{ }^{*}$ is obtained from the mobile device. Without the user's device, the adversary is unable

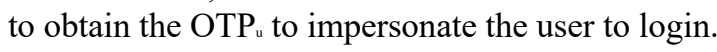

\subsection{Ensure Identity Privacy}

The session key is generated, $S K_{i}=h\left(I D_{i}\left\|S I D_{j}\right\| r_{i}\left\|r_{g}^{\prime}\right\| r_{j}^{\prime}\right)$ when mutual authentication is achieved during the $M^{\prime}{ }_{14} \stackrel{?}{=} h\left(M_{2} \| S K_{i}\right)$ is established upon receiving $\left\{M_{15}\right\}$ from GWN and $U_{i}$ computes it. A session key is an encryption and decryption key that allows continuous communication between the parties. This can secure the identity of $U_{i}$ as a new session key will be generated by random and it will be discarded after the session is ended. Thus, the use of session key will halt the attack from an adversary as each session comes with a random session key which then secures the user's identity (SearchSecurity, 2021).

\section{Complexity Analysis}

In order to facilitate the computational overhead comparisons, some notations are defined as follows. 


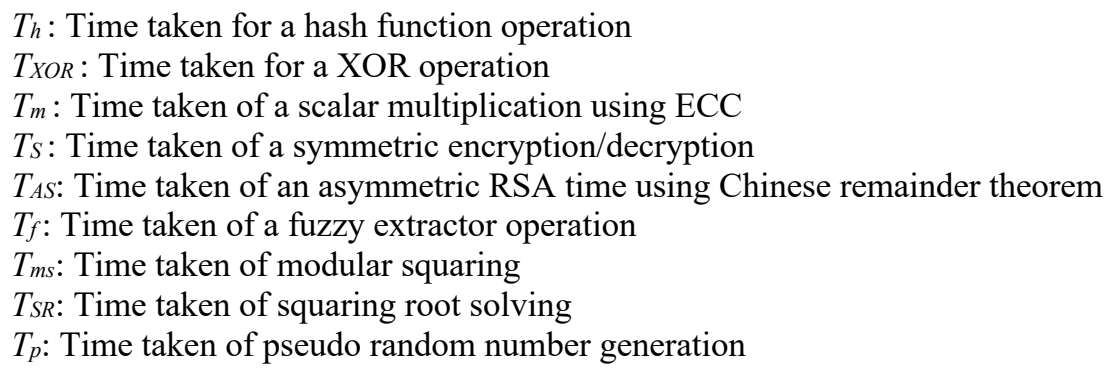

Usually, $T_{m}$ is larger than $T_{h}$ and $T_{S} . T_{h}$ is the same with the $T_{S}$. By using the simulation result of the Intel T5870 $2.00 \mathrm{GHz}$ experimental platform (Wang et al., 2015), we have $T_{m}=1.226 \mathrm{~ms}, T_{S}=0.0021 \mathrm{~ms}$ and $T_{h}=0.0026 \mathrm{~ms}$ respectively. By considering other schemes, we have $T_{A S}=12.06 \mathrm{~ms}$ (Feng, 2020), $T_{f}=63.075 \mathrm{~ms}$ (Banerjee et al., 2019), $T_{m s}=0.021 \mathrm{~ms}, T_{S R}=3.481 \mathrm{~ms}$ and $T_{p}=0.021 \mathrm{~ms}$ (Gope \& Sikdar, 2019) respectively. The bit-wise XOR operation $\left(T_{X O R}\right)$ is not included in this analysis because it is negligible as compared to other operations.

For the analysis of the authentication overhead, we assume 160 bits for the gateway's identity and sensor node's identity respectively. The user's identity length and timestamps are 80 and 32 bits respectively. Besides, the output of the hash function and random numbers are also 160 bits. It is supposed that the point on the elliptic curve $P=$ $\left(P_{x}, P_{y}\right)$ is 320 bits as $P_{x}$ and $P_{y}$ are $x$ and $y$ coordinates respectively which is 160 bits. ECC security remains the same because RSA public key length uses 1024 bits (Barker, 2018). The ciphertext block is 128 bits. We additionally suppose that the OTP length is 64-bits strings which will be shown as 8 decimal digits (Huang et al., 2013).

We compare authentication and computational overheads with related protocols during the authentication phase are shown in Table 2 and Table 3 (Feng, 2020) (Banerjee et al., 2019) (Sethia et al., 2018).

Table 2: Comparison of Computational Overhead among the proposed protocol and other schemes.

\begin{tabular}{|l|l|l|}
\hline Protocol & Total cost & Total time \\
\hline Sethia et al. & $46 T_{h}+258 T_{S}+26 T_{A S}$ & $314.21 m s$ \\
\hline Banerjee et al. & $31 T_{h}+2 T_{f}$ & $126.23 m s$ \\
\hline Feng & $8 T_{h}+2 T_{m s}+26 T_{S R}+2 T_{p}$ & $10.84 m s$ \\
\hline Our proposed protocol & $20 T_{h}+8 T_{S}+3 T_{m}$ & $3.746 m s$ \\
\hline
\end{tabular}

During the authentication phase, the computational overhead of the proposed protocol is $7 T_{h}+2 T_{S}+2 T_{m}$ for each user, $9 T_{h}+4 T_{S}+T_{m}$ for the gateway and $4 T_{h}+2 T_{S}$ for the sensor node. The total computational overhead is $20 T_{h}$ $+8 T_{S}+3 T_{m}=3.746 \mathrm{~ms}$. Table 2 summarizes the computational overhead for the compared schemes. The computational overhead of Sethia et al. (2018) protocol is the highest as they consider more about the RSA and AES encryption algorithms for asymmetric and symmetric encryption respectively. Banerjee et al. (2019) protocol is higher than Feng (2020) protocol because of the addition of the fuzzy extractor operator. It is clear that the proposed protocol is the lowest computational overhead as compared to that for other protocols. For the proposed protocol, the sensor node carries out symmetric encryption/decryption operations and hash function only.

Table 3: Comparison of Authentication Overhead among the proposed protocol and other schemes.

\begin{tabular}{|l|l|l|}
\hline Protocol & No of messages & No of bits \\
\hline Sethia et al. & 10 & 27936 \\
\hline Banerjee et al. & 3 & 2048 \\
\hline Feng & 7 & 3648 \\
\hline Our proposed protocol & 6 & 3360 \\
\hline
\end{tabular}

Table 3 shows the authentication overhead of all protocols. We can observe that the proposed protocol is lower than Sethia et al. (2018) protocol and Feng (2020) protocol but is higher than Banerjee et al. (2019) protocol. The total authentication overhead of the proposed protocol with six exchanged messages is 3360 bits. Sethia et al. (2018) protocol is the highest authentication overhead which requires 10 messages with around 27936 bits for communication since it uses much of the symmetric encryption. The proposed protocol is acceptable as compared to the overheads of the Banerjee et al. (2018) protocol since the proposed protocol has four factor authentication compared to three factor authentication of Banerjee et al. (2018) protocol. 


\section{Conclusion}

The Internet of Things (IoT) is a new technology that is predicted to revolutionize a variety of industrial sectors. The IoT allows physical devices worldwide to connect to the Internet by gathering and exchanging important and real-time data. As the number of devices rises, the computational cost associated with data transfer between devices and to the internet increases. In this paper, we have proposed an improved scheme of Li et al. scheme (2018) which is resist against spoofing attack and ensure identity privacy. The proposed scheme is to improve the protocol presented by Li et al. with a multi-factor authentication protocol by adding OTP once the user's biometric information is verified by the GWN. We have examined the security of our scheme and demonstrated that it is impervious to several well-known attacks such as impersonation attack, replay attack, and spoofing attack. Furthermore, complexity analysis that includes computational and authentication cost evaluations demonstrated that the enhancement of the proposed method is more reliable than existing protocols with lower cost in computational and presents reduction in power consumption significantly. IoT technology may be applied in a variety of sectors, including smart healthcare and smart grid, several security and privacy concerns are needed to be addressed. For example, in IoT-based smart healthcare, to create a authentication system that is lightweight for resource-restricted wearable devices, the aggregation of medical data for privacy protection and the publication of medical data protection remained as unresolved challenges. As for future work, researchers would need to further study and specifies the privacy and security needs for applications that are IoT-based and create solutions that are suited to overcome these challenges.

\section{Acknowledgements}

The authors would like to thank Faculty of Computer Science and Information Technology, Universiti Malaysia Sarawak to support this research work. This work is carried out as a short term research based class project.

\section{References}

Ahmad, Z., Khan, A. S., Shiang, C.W., Abdullah, J. \& Ahmad, F., Network intrusion detection system: A systematic study of machine learning and deep learning approaches, Transactions on Emerging Telecommunications Technologies, vol. 32 no.1, pp. e4150, 2021, 10.1002/ett.4150

Banerjee, S., Odelu, V., Das, A., Chattopadhyay, S., Rodrigues, J. J. \& Park, Y. (2019). Physically secure lightweight anonymous user authentication protocol for internet of things using physically unclonable functions. IEEE Access. 7, 85627-85644.

Balan, K., Khan, A.S., Julaihi, A.A., Tarmizi, S. \& Pillay, K.S., RSSI and Public Key Infrastructure based Secure Communication in Autonomous Vehicular Networks, International Journal of Advanced Computer Science and Applications (IJACSA) Volume 9 No 12 December 2018; pp. 298-304

Barker, E. (2018). Recommendation for Key Management. NIST, Gaithersburg, MD, USA. Tech. Rep.

Belcic, I. \& Farrier, E. (2021). What is spoofing and how can you prevent it? Retrieved from https://www.avast.com/c-spoofing

Cao, J., Yu, P., Ma, M. \& Gao, W. (2019). Fast authentication and data transfer scheme for massive NB-IoT devices in 3GPP 5G network. IEEE Internet Thing J, 6(2), 1561-1575.

Cao, J., Yu, P., Xiang, X.Y., Ma, M. \& Li, H. (2019). Anti-Quantum fast authentication and data transmission scheme for massive devices in 5G NB-IoT system. IEEE Internet of Things Journal, 6(6), 9794-9805. https:/doi.org/10.1109/JIOT.2019.2931724

Christensson, P. (2018, April). NFC Definition. Retrieved from https://techterms.com

Digital Guide Ionos. (2020). One-time password (OTP)-more security online. Retrieved from https://www.ionos.com/digitalguide/server/security/what-is-a-one-time-password-otp/

Dildar, M.S., Khan, N., Abdullah, J.B. \& Khan, A.S., Effective way to defend the hypervisor attacks in cloud computing, 2nd International Conference on Anti-Cyber Crimes (ICACC), pp. 154-159, 2017, 10.1109/Anti-Cybercrime.2017.7905282. 
Feng, Z. (2020). A Secure RFID Mutual Authentication Protocol for Healthcare Systems. Special Section on Lightweight Security and Provenance for Internet of Health Things, 8, 192192-192205.

Gope, P. \& Sikdar, B. (2019). Robust and Energy Efficient Authentication Protocol for Industrial Internet of Things. IEEE Internet of Things Journal, 6(1), 580-589.

Huang, Y., Huang, Z., Zhao, H. \& Lai, X. (2013). A new One-time Password Method. IERI Procedia, 4, 32-37

Imran, M.A., Mridha, M.F. \& Rahman, M. (2017). A Lightweight One Time Pad (OTP) and Biometric based Secure authentication scheme for IoT environment. Retrieved from https://www.researchgate.net/publication/320034057_A_Lightweight_One_Time_Pad_OTP_and_Biometr ic_based_Secure_Authentication_Scheme_for_IoT_Environment/stats

Ismail, K.A., Singh, M. M., Mustaffa, N., Keikhosrokiani, P. \& Zulkefi, Z. (2018). Security Strategies for Hindering Watering Hole Cyber Crime Attack. Procedia Computer Science, 127, 656-663.

Kaspersky. (2021). What is a Reply Attack? Retrieved from https://www.kaspersky.com/resourcecenter/definitions/replay-attack

Khan, A.S., Ahmad, Z., Abdullah, J. \& Ahmad, F. A Spectrogram Image-Based Network Anomaly Detection System Using Deep Convolutional Neural Network, IEEE Access, 2021, 9, 87079-87093.

Khan, N., Abdullah, J. \& Khan, A.S. Defending malicious script attacks using machine learning classifiers, Wireless Communications and Mobile Computing, vol. 2017; doi:10.1155/2017/5360472

Khan, A.S., Balan, K., Javed, Y., Abdullah, J. \& Tarmizi, S. Secure trust-based blockchain architecture to prevent attacks in VANET. Sensors (Switzerland), 2019, 19(22), 1.

Khan, A. S., Lenando, H., Abdullah, J. \& Fisal, N. Secure authentication and key management protocols for mobile multihop WiMAX networks. Jurnal Teknologi, 2015, 73(1), 75-81.

Khan, A.S., Javed, Y. \& Abdullah, J., Trust-based lightweight security protocol for device to device multihop cellular communication (TLwS), Journal of Ambient Intelligence and Humanized Computing, 2021, 10.1007/s12652-021-02968-6.

Li, J., Zhang, N., Chen, J. \& Du, R. (2020). Secure and Lightweight Authentication with Key Agreement for Smart Wearable Systems. IEEE Internet of Things Journal, 7(8), 7334-7344.

Li, X. Peng, J.Y., Liao, J. \& Choo, K.K.R. (2018). Robust and Energy Efficient Authentication Protocol for Industrial Internet of Things. IEEE Internet of Things Journal, 5(3), 1606-1615.

Ranger, S. (2020, February). What is the IoT? Everything you need to know about the Internet of Things right now. Retrieved from https:/www.zdnet.com/article/what-is-the-internet-of-things-everything-you-need-toknow-about-the-iot-right-now/

Maikol, S.O., Khan, A.S., Javed, Y. \& Bunsu, A.L.A., Petrus, C., A novel authentication and key agreement scheme for countering MITM and impersonation attack in medical facilities, International Journal of Integrated Engineering, vol. 13, no. 2, pp. 127-135, 2020.

Safkhani, M. \& Vasilakos, A. (2019). A new secure authentication protocol for telecare medicine information system and smart campus. IEEE Access, 7, 23514-23526.

SearchSecurity (2021). Session Key. Retrieved from https://searchsecurity.techtarget.com/definition/session-key

Sethia, D., Gupta, D. \& Saran, H. (2018). NFC secure element-based mutual authentication and attestation for IoT access. IEEE Transactions on Consumer Electronics, 64(4), 470-479.

Varanasi,P. (2020, October). Learn About Internal and External Cyber Attacks \& Ideas to be safe from them. CloudCodes. Retrieved from https://www.cloudcodes.com/blog/internal-external-cyber-attacks.html 
Wang, D., He, D., Wang, P. \& Chu, C. (2015). Anonymous Two-Factor Authentication in Distributed Systems: Certain Goals Are Beyond Attainment. IEEE Transactions on Dependable and Secure Computing, 12(4), 428-442. 10.1109/TDSC.2014.2355850. 